\title{
Creating Wireless Multi-hop Topologies on Space-Constrained Indoor Testbeds Through Noise Injection
}

\author{
Sanjit Krishnan Kaul, Marco Gruteser, and Ivan Seskar \\ WINLAB, Rutgers University \\ Rt 1 Tech Center, North Brunswick, NJ 08902-3390, USA \\ Email: \{sanjit,gruteser,seskar\}@winlab.rutgers.edu
}

\begin{abstract}
To evaluate routing protocols on a controlled indoor wireless testbed, the radio range must be compressed so that larger multi-hop topologies can be mapped into a laboratorysize area. We propose noise injection as a more flexible option than hardware attenuation and consider methods for mapping real world wireless network topologies onto the testbed. Our experimental results show that additive white Gaussian noise effectively reduces the radio range, without the need for hardware attenuation and careful shielding of wireless cards. We performed experiments for a free space propagation environment. By selecting node positions through an automated procedure, we were able to create a 5-node/4-hop string topology and a random partially connected 6-node topology in a $8 \mathrm{~m}$ by $8 \mathrm{~m}$ area with off-the-shelf IEEE 802.11 hardware.
\end{abstract}

\section{INTRODUCTION}

Wireless networking researchers increasingly emphasize experimental analysis of wireless systems and experimental validation of simulation models. Unfortunately, controlled experiments with wireless LAN systems are difficult and costly to conduct, primarily because experiment setups often require large distances between senders, receivers, and interferer. In particular, multi-hop topologies, where the network is not fully connected, require that the distance between some transmitterreceiver pairs is large enough to prevent direct communication. For example, off-the-shelf IEEE 802.11 radios have a transmission range of several hundred meters in a controlled line-ofsight propagation setting. While multi-hop wired testbeds can be set up in a small laboratory space, creating larger wireless multi-hop topologies, say with 5 hops, for experimentation with routing protocols would easily require a laboratory space of one kilometer length. Therefore, creating such topologies is usually only possible in outdoor settings with an uncontrollable radio propagation environment, which is affected by weather patterns, foliage, moving obstructions and other factors.

To facilitate controlled experimentation with larger wireless networks, researchers have frequently resorted to attenuating the transmission power [1]. While some off-the-shelve hardware provides transmission power control, the minimum setting is usually insufficient to create large network topologies in a laboratory space. ${ }^{1}$ Thus hardware attenuation is necessary, but inserting hardware attenuators requires manual labor and careful shielding for all nodes. Unless expensive variable attenuators are used, it also severely restricts experimenters flexibility in creating different topologies.

An alternative approach to attenuating transmission power is increasing the noise floor. Since the transmission range is primarily defined by a signal-to-noise ratio threshold, increasing noise has a similar effect on radio range as reducing transmission power. This approach is widely used in testing radio links. Lei and colleagues [2] have also applied it to an access point network with multiple clients. They introduced the radio mapping problem, which seeks to map realistic radio environments onto indoor testbeds through controlled noise injection. This can be achieved by changing sender, receiver, and interferer positions and their power levels until the communication channels between access point and each client meet their desired signal-to-noise ratio (SNR) constraint.

In this paper, we describe an experimental study to extend this approach to ad hoc networks with multiple senders and receivers. We analyze scenarios with a large number of candidate senders and receivers and a small number (four) of interferers. This means that the noise floor is not uniform across all receivers, but receivers will be affected differently based on the path loss from the interference sources to the receiver. This creates a challenging problem in selecting suitable nodes, so that the links between these nodes match the desired SNR constraints. While a WINLAB paper [2] shows that it is impossible to find an exact match for any arbitrary configuration, we present an algorithm that seeks to find an approximate match, if possible. Ultimately, we would like to recreate typical radio environments (e.g., home, office, urban) on a controlled indoor testbed. In this paper, we evaluate the algorithm by trying to recreate a radio environment that resembles those described by a free-space propagation model commonly used in wireless network simulations. This first step is already useful in providing an easier migration path for experimenters when porting their protocols and systems from a simulation

\footnotetext{
${ }^{1}$ For Atheros 5212-based IEEE 802.11 wireless cards the minimum setting is $1 \mathrm{~mW}$, which still results in a transmission range greater than $20 \mathrm{~m}$. Requiring the use of the minimum transmission power setting would also prevent experimentation with power control algorithms.
} 
environment to the more complex emulation environment of a testbed. Specifically, key contributions include:

- an automated mapping algorithm that selects suitable nodes based on packet error rate measurements.

- an experimental study of the effects of interference sources on the received signal strength, packet error rate, and symmetry of 802.11 channels.

- experimental evidence that 5-node/4-hop networks can be configured with only 4 interference sources in a $8 m$ by $8 m$ area.

The remainder of the paper is organized as follows. We begin by introducing controlled indoor wireless testbeds and the challenges in evaluating wireless protocols in section II. Section III defines the radio mapping problem and describes methods to map topologies onto a testbed. Section IV describes our experimental methodology for evaluation the effectiveness of noise generation and the mapping algorithms. The results are presented in section $\mathrm{V}$ before we conclude.

\section{Wireless Network Testbeds And the Radio MAPPING PROBLEM}

To evaluate novel network protocol ideas, researchers usually resort to a simulation environment where the complexity of underlying simulation models is manageable and results can be easily reproduced. Even on relative performance comparison of two protocols, say routing protocols, the underlying physical and MAC layer models can have a major impact. In the field of wireless networking, the art of choosing adequate simulation models is still being refined. Therefore, validation of simulation results on different wireless network testbeds is necessary to understand system performance.

\section{A. Controlled Indoor Testbeds}

We can distinguish between application-oriented testbeds and controlled indoor testbeds. Application-oriented testbeds are deployed in a realistic setting and may be used as a production network. Examples are MIT's Roofnet [3], or Dartmouths campus network [4]. These testbeds capture radio propagation effects of their deployment environment and tend to provide lower reproducibility of results because of a constantly changing radio environment. Controlled testbeds are tailored for earlier stage evaluation and are deployed in a shielded indoor environment to increase reproducibility of results.

One example of a controlled indoor testbed is provided by the Open Access Research Testbed for Next-Generation Wireless Networks (ORBIT) [5]. ORBIT's prototype indoor testbed comprises 128 IEEE $802.11 \mathrm{a} / \mathrm{b} / \mathrm{g}$ radio interfaces attached to 64 static nodes arranged on an 8 by 8 grid, as shown by Fig. 1 . The antennas are mounted on the sides of crates, at 45 and 225 degree positions when looking at the topside of a node and are connected through shielded cables to the Atheros-based wireless cards. Every node is a small form factor PC with $1 \mathrm{GHz}$ Via C3 CPU, 512 MB RAM, 20 GB hard disk and three ethernet ports, one of which is used for node configuration and control.

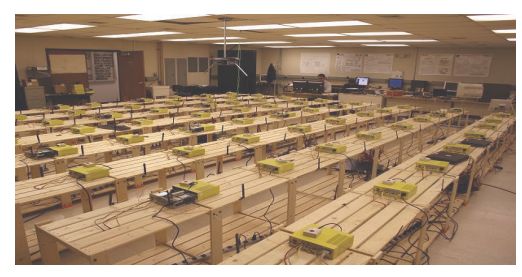

Fig. 1. The prototype ORBIT indoor testbed comprises 64 nodes (small form factor PCs) arranged on a grid.

\section{B. Radio Mapping Goals}

One foreseeable use that requires multi-hop topologies is the emulation of ad-hoc networks on indoor testbeds. As these testbeds can only be of limited size, mere pathloss is not sufficient to create multi-hop topologies where some nodes cannot directly communicate with each other. The radio environment is also insufficiently complex to understand protocol performance under real-world conditions. For example, multipath and attenuation due to moving obstructions and time-varying interference are absent.

This raises the question of which radio propagation characteristics to emulate on an indoor testbed. These characteristics vary widely over different radio environments (e.g., indoor vs urban setting) and experimenters might use the testbed with different goals in mind. The reasons for moving from a simulation to a testbed environment could be to validate and improve simulation models for physical and MAC layer, to experiment with detailed physical and MAC layer effects that would consume too much computational time in a standard network simulator, or even to test and debug a system prototype implementation under varying conditions (while much of the code base can be tested in simulation, interfaces with the OS kernel usually differ and must be debugged in a testbed setting).

In many cases, such as the study of routing protocols, it may be desirable to recreate the packet error patterns of a realistic example radio environment based on available packet trace data. For example, data from urban outdoor measurements [3] or home environment measurements [6] could be used. When first migrating simulation code to a testbed environment, however, researchers may prefer a simplified propagation environment that resembles the simulation models. This allows a direct comparison of results and eases debugging because it isolates the effects of radio propagation patterns from the effects of protocol differences in simulation and testbed.

Thus, we believe it is best to provide a flexible system, where the propagation characteristics can be changed in between experiments. This work provides a first step towards that goal. We concentrate on creating scenarios without timevarying channel conditions and evaluate the system by creating a multi-hop radio propagation environment that matches a freespace propagation model. The free-space propagation model is a commonly used model in the $n s-2$ network simulator as part of the CMU Monarch and ISI extensions [7], for example. It assumes that there is a line-of-sight path between transmitter 
and receiver. It calculates path loss solely as a function of distance. The received signal power $P_{r}$ at distance $d$ from the transmitter equates to

$$
P_{r}(d)=\frac{P_{t} G_{t} G_{r} \lambda^{2}}{(4 \pi)^{2} d^{2} L}
$$

where $P_{t}$ is the transmitted signal power, $\lambda$ is the wavelength, $L$ is the system loss, and $G_{t}$ and $G_{R}$ are the transmitter and receiver antenna gains, respectively. The model is further simplified to $P_{r}(d)=\frac{P_{t} \lambda^{2}}{(4 \pi)^{2} d^{2}}$ in many simulations. We have chosen the free-space model as a test case for evaluating our mapping algorithms because it is a well-understood reference model without time-varying channel characteristics.

\section{Related Work}

The most common solution to address the spatial scaling problem of wireless testbeds is through attenuation at the transmitter. Sanghani [8] and colleagues have built a testbed with heavily attenuated antennas that can be repositioned to create different topologies. This approach requires careful shielding, which can be very time consuming and expensive to install. Producing a reliable setup for large numbers of radios (for example the full ORBIT deployment will contain 800 radios), with hacks using aluminum foil would be very difficult. Using a precision RF enclosure instead would add substantial pernode costs. Another option is to still use attenuation but instead of antennas connect the radios by wire and control topology through resistive splitters and combiners, as implemented by Kaba and Raichle [1]. In this case, spurious signal leakage, is less of a concern but attenuators (and especially splitters and combiners) must still be manually configured for every experiment (the cost of connecting hundreds of radios to electronically controlled variable attenuators, splitters, and combiners would be prohibitive). In our work, we explore the alternative of noise injection for large-scale testbeds, which does not require shielding and promises the flexibility to create a wide range of possible topologies. Controlling noise levels can also be fully automated, manual reconfiguration is unlikely to be required. While our current setup relies on an expensive signal generator, we expect that specialized additive white gaussian noise sources can be added for substantially less cost than RF enclosures.

The concept of introducing additive white Gaussian noise to emulate path loss is a standard technique to evaluate communication system performance between a single sender and receiver. For example, Pajukovski and Savusalo [9] present experimental results for a CDMA system that were obtained by injecting AWGN into the channel.

The area of applying these techniques to large-scale multiple sender and receiver scenarios is relatively unexplored. Lei and colleagues [2] described a method to emulate the signal-tonoise ratio for several clients associated to an access point. This work, to our knowledge, first introduced the link mapping problem and described an interference solution for wireless indoor testbeds. It evaluated the feasibility of mapping several access point to client links based on a simulation study. We extend this work to multi-hop, bidirectional links and present proof-of-concept experimental results.

Other testbeds have been built and experiments conducted without scaling the communication range. These experiments range from a group of students carrying laptops on a football field or in a larger building [10], [11] over experiments with radios in vehicles (e.g., [12], [13]) to in-city deployments of mesh networks [3]. These deployments substantially differ from a stationary indoor testbed in the degree of reproducibility of results. The indoor testbed is not affected by sporadic external interference, foliage, moving obstructions, weather conditions, and other parameters. Thus, it provides a more convenient environment for detailed scientific study before systems are evaluated in a less predictable outdoor environment.

\section{RAdio MAPPING Methods}

We assume that an experimenter has access to a number of interferers and a number of nodes that can act as senders and receivers. The interferers are configured to emit additive white Gaussian noise (AWGN) with an experimenter-specified center frequency, bandwidth, and power level. The experimenter can adjust the positions of both the interferers and nodes within a square area (either by physically rearranging nodes or, if working with a stationary testbed, the experimenter may select nodes close to the intended positions from a larger number of candidates). The interference levels are configurable at the interferers and can be used to vary the link conditions between any two nodes. The experimenter can also control bitrate and transmission power on the senders.

We define the radio mapping problem as follows: Given a virtual scenario with a set of $n$ nodes and a time-invariant virtual packet error rate on every link between two nodes, configure the testbed so that the packet error rate (PER) on the links between the chosen testbed nodes approximates the PER in the virtual scenario (note that we do not consider packet collisions when we refer to PER). We choose the packet error rate as a link metric because it is a key parameter that affects the performance of higher layer protocols and it can be easily measured on the testbed (measuring SNR in comparison is more difficult, since the implementation and calibration differs between different card models). The desired link status can be captured in a link matrix that defines the PER on each unidirectional link between a given sender and receiver. The packet error rates lie in the range of $0 \%$ to $100 \%$.

For example, let us model a free space scenario. Packet error rates for the free space propagation model are computed based on distance. Given the transmission power $P_{t}$ and the distance $d$ between two nodes, a packet is received if the received power $P_{r}$ is greater or equal to a threshold $P_{t h}$, which corresponds to the receiver sensitivity. Thus, the free space propagation model defines a hard distance threshold before which all and after which no packets are received. In other words, each link will be assigned a packet error rate of either $0 \%$ or $100 \%$ in stationary networks. We will also refer to these values as link up and link down, respectively. Table I shows a 
sample link matrix for a four node string topology assuming a free space propagation model. The matrix in the particular case is symmetric, as the free space model can only generate symmetric links. The goal of the radio mapping methods then is to identify a set of nodes, and an interference configuration that approximates a given link matrix. This sample link matrix assumes the same packet size and PHY rate on all the links. It could easily be further generalized by adding packet size and PHY rate parameters for each link.

\begin{tabular}{|c||c|c|c|c|}
\hline & 1 & 2 & 3 & 4 \\
\hline \hline 1 & & $0 \%$ (up) & $100 \%$ (down) & $100 \%$ (down) \\
\hline 2 & $0 \%$ (up) & & $0 \%$ (up) & $100 \%$ (down) \\
\hline 3 & $100 \%$ (down) & $0 \%$ (up) & & $0 \%$ (up) \\
\hline 4 & $100 \%$ (down) & $100 \%$ (down) & $0 \%$ (up) & \\
\hline
\end{tabular}

TABLE I

SAMPLE LINK MATRIX TABLE FOR FOUR NODES IN A STRING TOPOLOGY.

We consider an automated Select Nodes with Fixed Interference (SNFI) procedure and compare it against a manual Select Interference for Fixed Nodes (SIFN) procedure as a baseline.

The SNFI algorithm measures link quality (in terms of PER) for each directional link between any available testbed nodes and an exhaustive search through this link quality graph yields the nodes that best approximate the virtual scenario. It accepts as input the desired link status matrix, the number of nodes in this matrix, and a PER allowance which allows defining a range of acceptable PER values given by (PER specified in link matrix \pm perAllowance).

Interferers are placed at fixed positions close to the corners of the square with an interference power setting that maximizes the dynamic range on the grid. Algorithm box 1 provides a detailed description of SNFI. It begins by capturing a snapshot of the grid as seen by each node. The snapshot is defined as the one hop connectivity between the transmitting node and every other node. We measure the one-hop connectivity in terms of PER. The algorithm then searches a set of nodes that match the desired link status map and reports the first match. Searching is implemented using a backtracking approach. The algorithm may not always find a match, in this case it can be restarted with a higher perAllowance parameter.

We define the baseline SIFN approach only for the freespace propagation model. In this case the algorithm selects nodes on the testbed so that the relative distances between the nodes (their antennas) best approximate the relative distances between them in the desired free-space scenario. Interferers are then placed next to the node antennas. The algorithm starts by emitting the same power level from all interferers and measures link quality on all links. It then increases the interference in steps until all links marked down in the link status matrix show sufficiently high PER. If this leads to high PER on any of the links that are marked up, it selectively reduces interference power of the antenna next to the receiver node of this link.

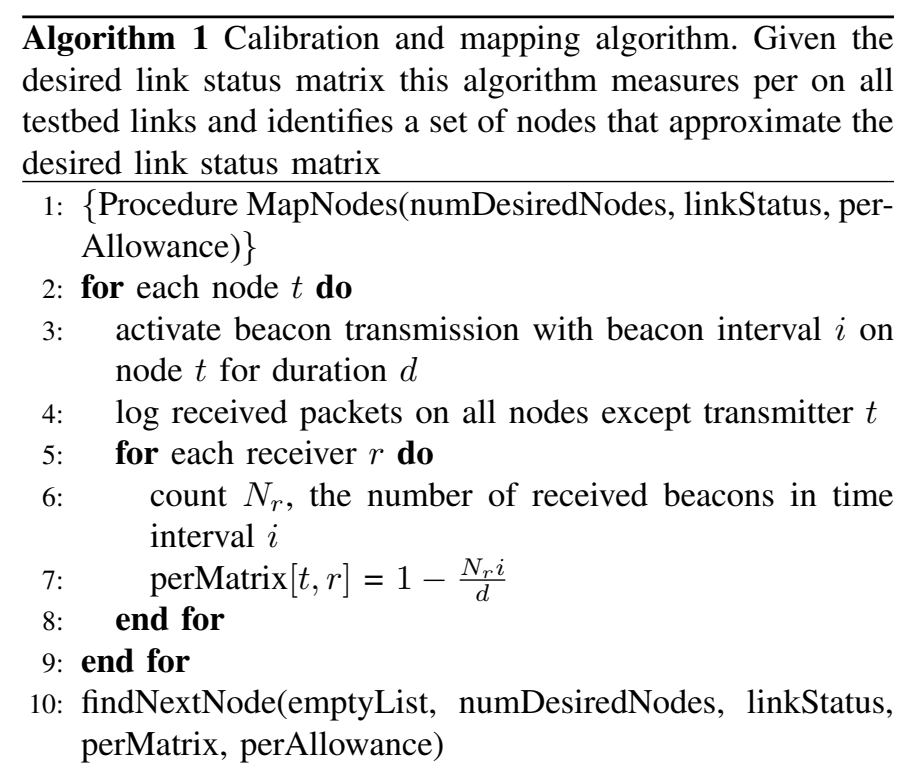

\{Procedure findNextNode(nodeList, numDesiredNodes, linkStatus, perMatrix, perAllowance) $\}$

11: for all nodes $n$ do

12: $\quad$ if $n$ in selectedNodeList then

13: $\quad$ continue with next node

14: else

15: if perMatch(selectedNodeList, $\mathrm{n}$, linkStatus, perMatrix, perAllowance) then add $n$ to selectedNodeList

16: if length of selectedNodeList $=$ numDesiredNodes then output selectedNodeList exit

else

findNextNode(selectedNodeList, numDesiredNodes, linkStatus, perMatrix, perAllowance)

22: $\quad$ end if

23: $\quad$ remove last node from selectedNodeList

24: $\quad$ end if

25: end if

26: end for

\{Procedure perMatch(nodeList, node, linkStatus, perMatrix, perAllowance) $\}$

$\left\{\operatorname{minPERAllowed}_{i j}=\operatorname{perMatrix}[i, j]-\operatorname{perAllowance}\right\}$

$\left\{\operatorname{maxPERAllowed}_{i j}=\operatorname{perMatrix}[i, j]+\right.$ perAllowance $\}$

27: for each node $n$ in nodeList do

28: if (linkStatus $[n$, node $]>=\operatorname{minPERAllowed}_{n, \text { node }}$ ) AND (linkStatus $[n$, node $]<=\operatorname{maxPERAllowed~}_{n, \text { node }}$ ) AND (linkStatus $[$ node,$n]>=\operatorname{minPERAllowed}_{\text {node }, n}$ ) AND (linkStatus $[$ node,$n]<=\operatorname{maxPERAllowed}_{\text {node }, n}$ ) then

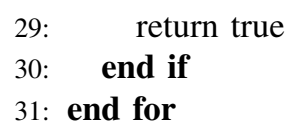




\section{EXPERIMENT SETUP}

The ORBIT grid used is a 8 by 8 square grid consisting of 64 radio nodes, 32 of them fitted with two Atheros 5212-based IEEE $802.11 \mathrm{a} / \mathrm{b} / \mathrm{g}$ cards each. The remaining are Intel cards. We use Atheros cards for all our experiments as we found the drivers more open and malleable than others. Let us refer to each node as node $(x, y)$, where $x$ is the row index and $y$ is the column index (both in the interval $[1,8]$ ). The Atheros and Intel-based nodes are placed alternately on the grid-thus every other node is unused in our experiments. Adjacent nodes are separated by a distance of $3 \mathrm{ft}$.

Each node runs a Debian Linux distribution with 2.4.26 kernel and uses the madwifi stripped driver. ${ }^{2}$ This allows generation and processing of raw IEEE802.11 encapsulated frames from the Click Modular Router [14]. One node is configured in Master mode to transmit 802.11 beacon packets, while all other nodes act as receivers. Thus packet error due to collisions is not a possibility. The receivers' driver provides all received MAC frames encapsulated with a so-called Prism header that contains bitrate, received signal strength indicator (RSSI), and other physical layer information. A Click script on the receiver extracts and $\operatorname{logs}$ the sequence number and RSSI for each correctly received frame.

We implemented the SNFI algorithm in a Perl script, that executes on a server and can remotely execute commands on the nodes through ssh. Log files were copied back to the server and the packet error rate (PER) at each receiver node is calculated as $1-\frac{N_{r}}{N_{t}}$, where $N_{r}$ is the number of packets in the $\log$ file and $N_{t}$ is the number of transmitted beacons. Since the transmitter sends one beacon per $100 \mathrm{~ms}, N_{t}=\frac{d}{100 \mathrm{~ms}}$, where $\mathrm{d}$ is the duration of the experiment in milliseconds.

The testbed supports additive white Gaussian noise interference generation at center frequencies of $250 \mathrm{KHz}$ to $6 \mathrm{GHz}$. An Agilent E4438C ESG vector signal generator provides the interference signal. The signal generator is connected to four omni-directional noise antennae, placed between node $(2,1)$ and node $(2,2)$; node $(2,7)$ and node $(2,8)$; node $(7,1)$ and node $(7,2)$; and node $(7,7)$ and node $(7,8)$. The noise power is split equally amongst the noise antennae. An amplifier is used to amplify the signal from the ESG before it is split amongst the noise antennae. The amplifier approximately compensates for any losses in the coaxial cables that connect the ESG to the antennae. All experiments carried out used I/Q modulated AWGN as the interference. Noise power can be varied between $-95 \mathrm{dbm}$ and $-5 \mathrm{dbm}$ (at a granularity of $0.5 \mathrm{dbm}$ ), and distributed over a noise bandwidth of up to $40 \mathrm{MHz}$

Figure 1 shows the ESG and the antenna placed between node $(2,1)$ and node $(2,2)$. Unless otherwise mentioned the wireless cards and the noise generator use the configuration shown in table II. We selected the highest transmit power and

\footnotetext{
${ }^{2}$ Available from http://www.pdos.lcs.mit.edu/ jbicket/madwifi.stripped/. The madwifi stripped driver is a fork from the regular madwifi driver that provides integration with Click. It also provides a pseudo-ibss mode that we used for our experiments because the ad-hoc mode implementation in the standard madwifi driver is not stable.
}

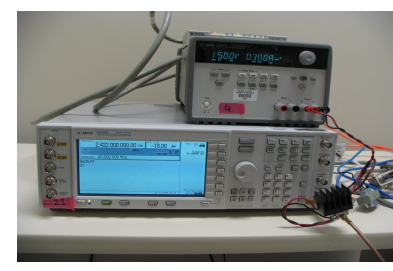

(a)

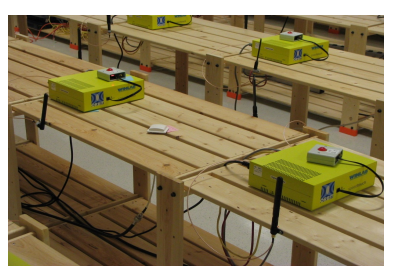

(b)
Fig. 2. (a) The ESG vector signal generator with the amplifier and power supply. (b) The noise antenna (white square) placed between node $(2,1)$ and node $(2,2)$.

lowest available bitrate for these experiments, because they result in largest possible transmission range and so present the most challenging scenario for our approach.

\begin{tabular}{|l|l|}
\hline Parameter & Default Setting \\
\hline \hline Interference Type & AWGN \\
\hline Interference Power & $-5 \mathrm{dbm}$ \\
\hline Interference Bandwidth & $20 \mathrm{MHz}$ \\
\hline Interference Center Frequency & $2.422 \mathrm{GHz}$ \\
\hline Channel & 3 \\
\hline Mode & $802.11 \mathrm{~g}$ \\
\hline Transmit Power & $20 \mathrm{dbm}$ \\
\hline PHY rate & $1 \mathrm{Mbps}$ \\
\hline
\end{tabular}

TABLE II

DEFAULT EXPERIMENT PARAMETERS

Unless otherwise mentioned PER was measured over a period of $30 \mathrm{sec}$. We experimentally established that measurement variance only marginally decreases over longer intervals. All measurements show packet losses uniformly distributed across the entire measurement period-no packet loss bursts are observed.

\section{RESUlts}

The experiments conducted address the following questions:

- How does the injected AWGN affect packet error rate and received signal strength on the testbed?

- How reproducible are specific noise conditions?

- How large are the multi-hop topologies that can be constructed, if any?

\section{A. Effect of Noise Sources on PER and RSSI}

Figure 3 shows the packet error rate observed at all nodes for node $(1,4)$ as transmitter and the noise set to $-5 \mathrm{dbm}$. The transmitter transmits beacons for a period of 5 mins $(3000$ beacon packets). As is seen from the plot, there are two dominant receiver behaviors: full packet loss (100\% PER) and no packet loss ( $0 \%$ PER). A few nodes also show partial packet loss. As expected, the nodes receiving all packets tend to be closer to the transmitter than the others. The correlation with distance from transmitter and noise source is not strong for all nodes, this is probably due to the specific propagation environment of the prototype setup. 


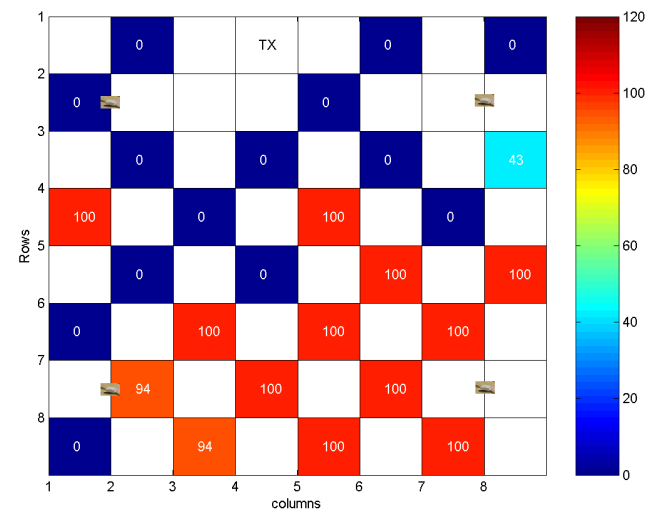

Fig. 3. Percentage Packet Loss with node $(1,4)$ as Tx and other nodes as receivers but for the nodes that are in white. Noise is set at $-5 \mathrm{dbm}$. The two mini white rectangles in row 2 and row 7 are the four noise antennae placed between node $(2,1)$, node $(2,2)$; node $(2,7)$, node $(2,8)$; node $(7,1)$, node $(7,2)$; node $(7,7)$, node $(7,8)$ respectively.

Figure 4 shows a boxplot of the correlation between the RSSI and the PER observed by a receiver. Each sample contains a mean RSSI value of all packets received by one receiver within the experiment duration and the receivers packet error rate over this interval (since RSSI is only provided for received packets, samples cannot be obtained for nodes that receive no packets). Such RSSI-PER pairs were collected from transmissions of 27 senders for interference levels $-20 \mathrm{dbm}$ to $0 \mathrm{dbm}$ in increments of $5 \mathrm{dbm}^{3}$. The graph shows higher packet error rates and higher variance for lower RSSI values. This indicates that for the Atheros-based cards RSSI can be interpreted as the signal-to-noise ratio observed by the receiver, rather than the signal power.

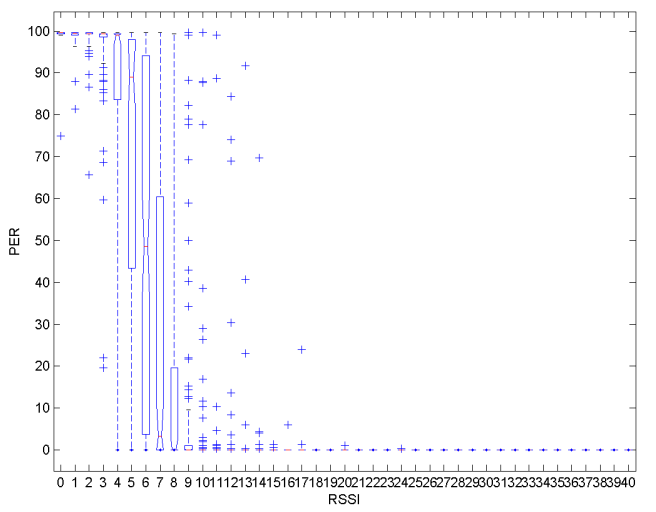

Fig. 4. RSSI PER correlation

The next two figures further show the spatial distribution of the effect of noise across different noise levels. Figure 5 shows the average RSSI received at various nodes for node $(1,4)$ as transmitter. The RSSI are plotted for noise levels

\footnotetext{
${ }^{3} 27$ transmitters, 26 receivers per transmission, 5 noise levels
}

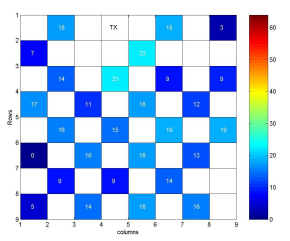

(a)

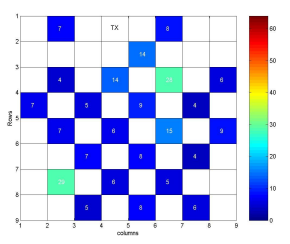

(c)

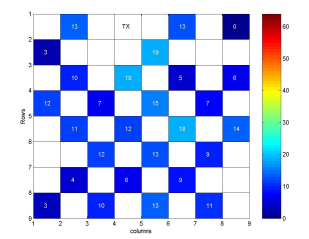

(b)

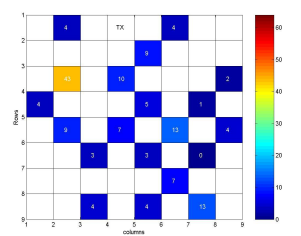

(d)
Fig. 5. Average RSSI measured at Atheros Nodes with node $(1,4)$ as transmitter for different interference levels. Atheros outputs RSSI as integers in $[0,64]$. Figures (a) $-15 \mathrm{dbm}$ (b) $-10 \mathrm{dbm}$ (c) $-5 \mathrm{dbm}$ (d) 0dbm. RSSI values are not plotted for nodes not a part of the experiment or those who did not receive any packet during the experiment i.e., the nodes with $100 \%$ PER in Figure 6.

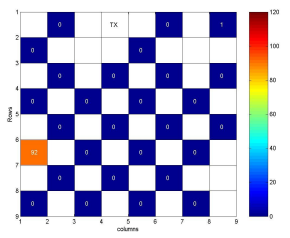

(a)

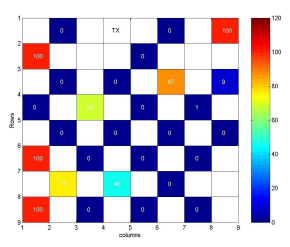

(c)

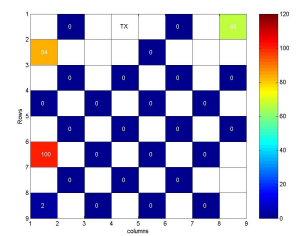

(b)

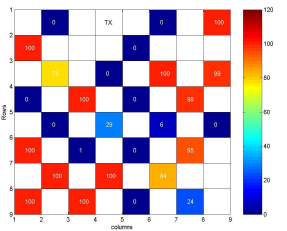

(d)
Fig. 6. Average PER measured at Atheros Nodes with node $(1,4)$ as transmitter for different interference levels. Figures (a) $-15 \mathrm{dbm}$ (b) $-10 \mathrm{dbm}$ (c) $-5 \mathrm{dbm}$ (d) $0 \mathrm{dbm}$. For the same experiment the RSSI values are shown in Figure 5

of $-15 \mathrm{dbm},-10 \mathrm{dbm},-5 \mathrm{dbm}$ and $0 \mathrm{dbm}$. The corresponding PER is plotted in Figure 6. Nodes close to the noise antennas (corners) and farther from the transmitter tend to show higher packet error rates, although the effect varies across trials (e.g., compare with Figure 3).

Figure 7 and Figure 8 report on the variation in link asymmetry with increasing interference power ( $-20 \mathrm{dbm}$ to $0 \mathrm{dbm}$ ). The link asymmetry is shown in terms of PER in Figure 7 and in terms of RSSI in Figure 8. In terms of PER the asymmetry is defined as the difference in PER of the forward link and the reverse link between a node pair. Similarly, in terms of RSSI link asymmetry is defined as the difference in the RSSI of the forward and reverse links between any two nodes. As both the plots illustrate, link asymmetry increases for higher interference levels. The plot summarizes asymmetry for all 
links at the different noise levels. The placement of the noise antennae as shown in Figure 3 will lead to asymmetric links on the grid when interference is introduced as the nodes differ in their distance from the noise antennae. The asymmetry can be explained by the different interference levels encountered by a node pair, for example node $(2,1)$ and node $(4,3)$ on the grid. Consider the bi-directional link between node $(2,1)$ and node $(4,3)$. Node $(2,1)$ is closer to a noise antenna. Hence it is more affected by interference than node $(4,3)$. The link node $(2,1)$ $>$ node $(4,3)$ (node $(2,1)$ transmitting and node $(4,3)$ receiving) will show a higher PER than node $(4,3)$ - $>$ node $(2,1)$ as it is the interference at the receiver that determines the PER of a link. The ability to produce asymmetric links on the testbed can be used to emulate asymmetric links that are found in real world scenarios, for example in the home wireless environment [6].

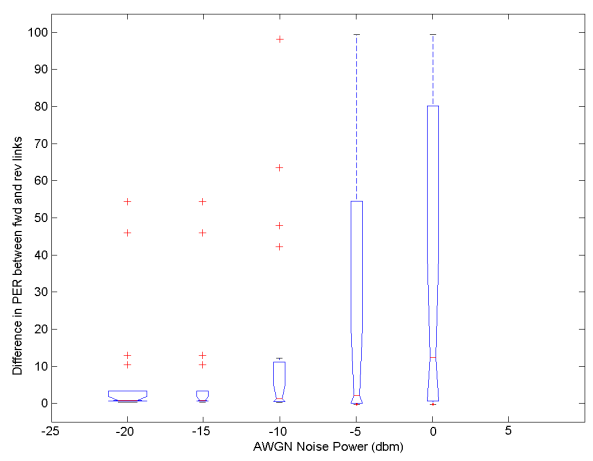

Fig. 7. Asymmetry of Links as difference in PER of fwd and rev links

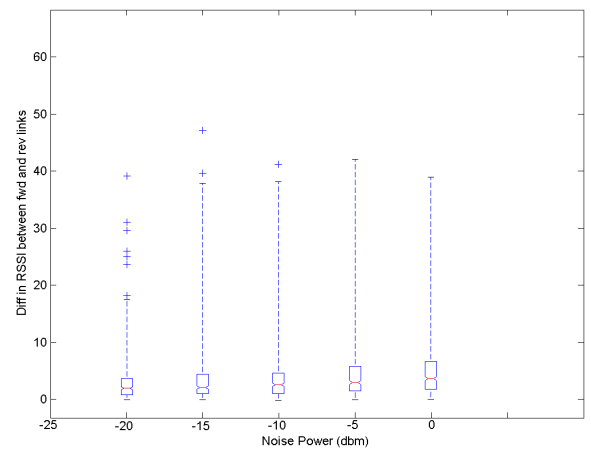

Fig. 8. Asymmetry of Links as difference in RSSI of fwd and rev links

\section{B. Reproducibility}

Figure 9 shows the results of the same experiment as reported in Figure 3 when repeated on the next day. While most of the nodes show consistent behavior across the two trials, the PERs for nodes $(7,2),(8,3)$, and $(3,8)$ exhibit large differences.

Figure 10 highlights the spatial distribution of the 10 out of 32 nodes with high variance in PER across 16 trials of the experiment (a trial's result plotted in Figure 3) over a two

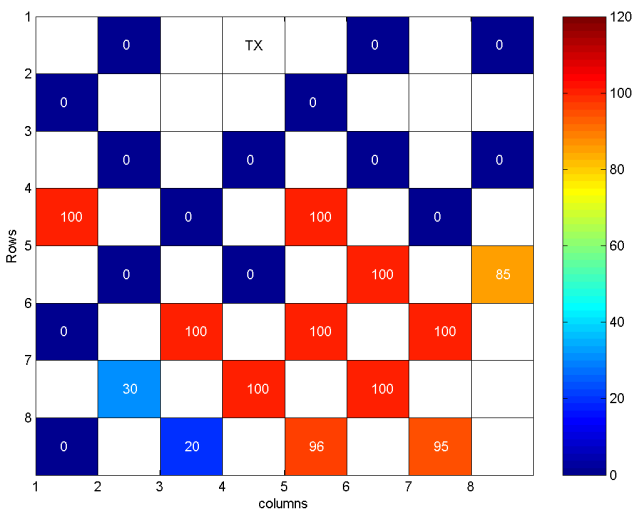

Fig. 9. Another trial of the same experiment as in Figure 3 carried out on the next day. Percentage Packet Loss measured with node $(1,4)$ as Tx and other nodes as receivers but for the nodes that are in white. Interference is set at $-5 \mathrm{dbm}$ as for results obtained in Figure 3.

week period. Nodes in dark black are the ones that show high variance in PER over the two week period. The maximum difference in PER observed at any node across these trials was $70 \%$. The high variance nodes affect the repeatibility of topologies mapped on the testbed. The dark black nodes are seen to have marginal PERs over some or all of the 16 trials (two are plotted in Figure 3 and Figure 9). For example, the nodes $(7,2),(8,3),(8,5),(8,7),(5,8)$ show marginal PERs in Figure 9 .

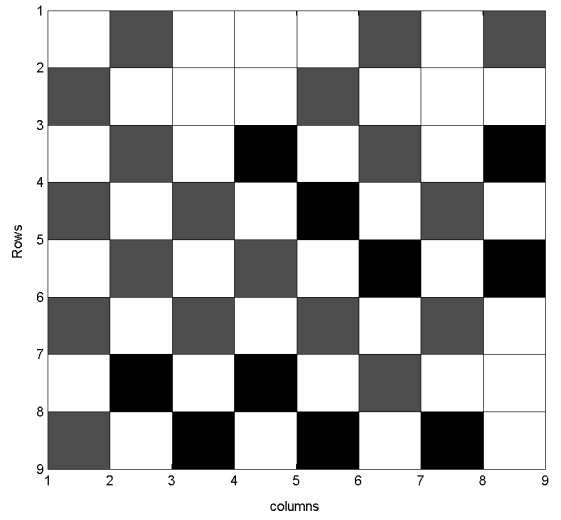

Fig. 10. Spatial distribution of nodes with high PER variance across 16 trials carried out over a two week period. PER obtained at the nodes in two of the 16 trials is plotted in Figure 3 and Figure 9. The transmitter is located at node $(1,4)$ for all the trials. The nodes in dark black are the ones showing high variance in PER.

Figure 11 shows packet error rates observed by node $(7,4)$ at different interference levels from $-25 \mathrm{dbm}$ to $4 \mathrm{dbm}$ (note that the interference level is set at the signal generator and does not represent the exact interference power observed by the receiver). Each curve represents a single trial of the experiment with packet error rates normalized to unity. The five trials were carried out on five consecutive days. A steep increase in packet losses is seen for interference levels between $-8 \mathrm{dbm}$ 
and $0 \mathrm{dbm}$. Within this region, a small change in interference power can generate a large difference in packet error rate.

The curves for different trials are slightly offset from each other. For example, the packet error rate for an interference level of $-5 \mathrm{dBm}$ ranges from $10 \%$ to $80 \%$ across trials. This graph explains why large difference in packet error rates are observed on some nodes (the dark black nodes in Figure 10), while other nodes show hardly any variance. The nodes with high variance in PER are operating with SNR levels close to their minimum sensitivity, thus small changes in environmental conditions may cause large changes in PERs.

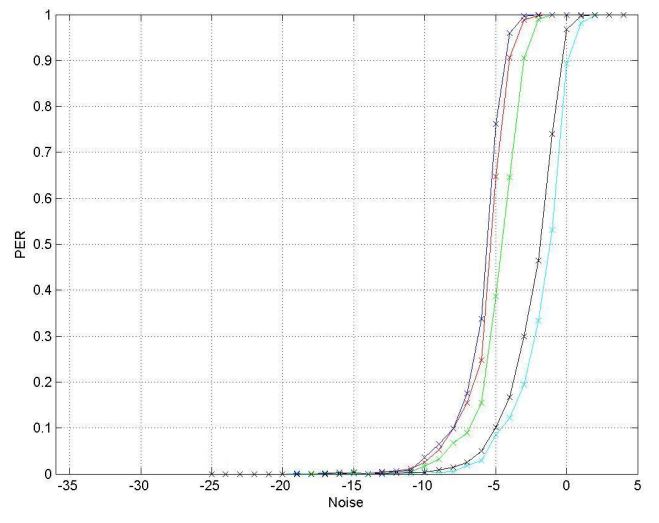

Fig. 11. Packet Error Rates at node $(7,4)$ for noise varied between $-25 \mathrm{dbm}$ and $4 \mathrm{dbm}$ at a granularity of $1 \mathrm{dbm}$. The Tx is node $(1,4)$. The five differently colored lines correspond to five different repetitions of the experiment on different days. Observe the steep increase in the packet losses as the noise generator setting changes from $-8 \mathrm{dbm}$ to $0 \mathrm{dbm}$.

Figure 12 shows the changes in PER over time relative to per-node mean. The per-node mean is the mean PER observed at a node over different trials of an experiment. The $\mathrm{x}$-axis shows the trial index-results were collected for two trials per day over a period of eight days. Each point represents the packet error rate observed at one receiver, measured relative to the mean for this receiver across all trials (the per-node mean for the receiver). No clear trend can be observed over time. During each trial some nodes show higher PER, while others show less PER than usual. External interference is likely to affect all nodes in a similar way, thus this explanation can be ruled out. We observed similar behavior for other transmitters, although the nodes that exhibit high variance change with the position of the transmitter. Thus, these variations are also unlikely to be caused by variations in transmission power.

\section{Feasibility of Multi-hop Topologies}

We first manually executed the SIFN method to determine the feasibility of mapping a 4-node, 3-hop string topology onto the testbed. We selected nodes $(1,2),(1,4),(1,6)$, and $(1,8)$, which are equally spaced and placed along an imaginary straight line. After placing noise antennas next to the node antennas, we found the desired topology at an interference level of $-10 \mathrm{dbm}$. We also moved the noise antenna besides node $(1,2)$ by approximately $7 \mathrm{cms}$ in a direction perpendicular

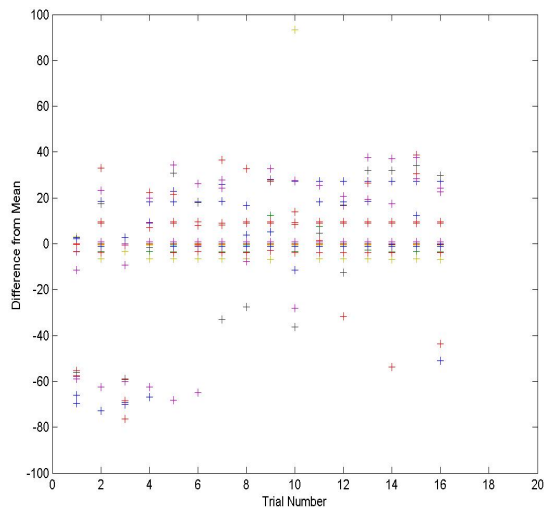

Fig. 12. Packet error rate, plotted as difference from mean, for a total of 16 trials with node $(1,4)$ as Tx and other nodes as receivers. Noise is set at $-5 \mathrm{dbm}$. For a trial number, each ' + ' corresponds to a different receiver node.

to the line connecting the nodes, because the noise level was too high at this node. To verify the multi-hop path we ran a ping session over the AODV routing protocol for a period of 20 mins. Ping showed $2 \%$ packet loss and an average delay of $89.8 \mathrm{~ms}$. During this experiment we consistently saw three hops between the participating nodes. Figure 13 shows the link packet error rates for this setup, measured as described in section IV. This configuration creates a near-perfect mapping of a free-space 4 node string topology.

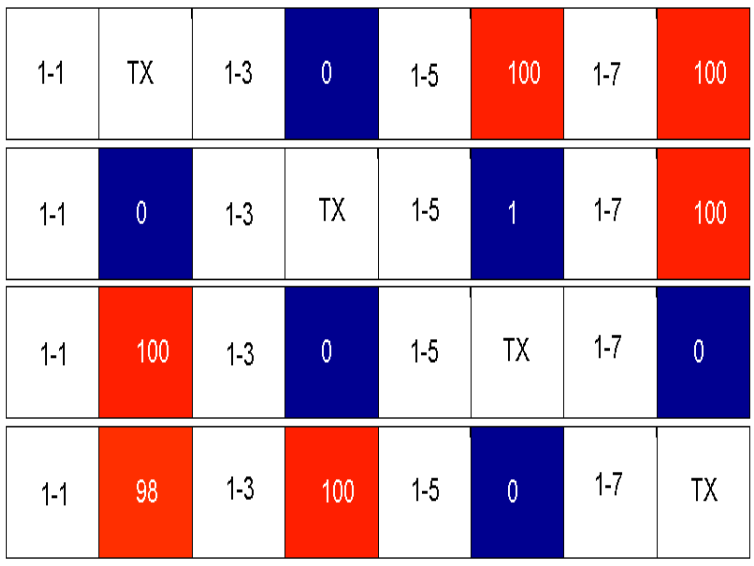

Fig. 13. Three hop experiment PER plot

Figure 14 shows that the automated SNFI algorithm with fixed noise antenna positions (as described in section IV) can also identify mappings for 3-hop string topologies. We assume a free space propagation model for experimental purposes. In a free space model a link may be either up (0\% PER) or down (100\% PER). Also, all links are symmetric. The graph shows the number of node mappings identified by the algorithm for different string-topology lengths, noise levels, and perAllowance parameters. The noise levels were set to $-10,-5$ and $0 \mathrm{dbm}$ and the perAllowance parameter to 5,10 , and $15 \%$ (which corresponds to a maximum PER of 5, 10, 
and $15 \%$ for a link that should be up and a minimum PER of $95 \%, 90 \%$, and $85 \%$ for a link that should be down) for each noise level. The number of two hops peaks at $-5 \mathrm{dbm}$. While the higher 0dbm noise level shows fewer 2-hop links it creates a few 3-hop and a couple of 4-hop links. For example, one of the three hop links created was that between node $(2,1)$, node $(4,1)$, node $(8,3)$ and node $(8,7)$. Referring back to figure 3 , it can be seen that the noise antennae are very close to the nodes $(2,1),(8,3)$ and $(8,7)$ (the noise antennae are the ones between node $(2,1)$ and $(2,3)$, node $(7,1)$ and $(7,2)$, node $(7,7)$ and $(7,8)$, respectively). Thus this setup shows similarities to the manually created configuration. The experiment was repeated on three different days. The total number of string topologies found were similar to the reported numbers. The number of topologies obtained on different days varied slightly. For example, on the second repetition four 4-hop topologies were found for a noise setting of $0 \mathrm{dbm}$ and perAllowance of $5 \%$ as against the two 4-hop topologies plotted in Figure 14. Reproducibility may, however, be improved by slightly adjusting the interference power level.

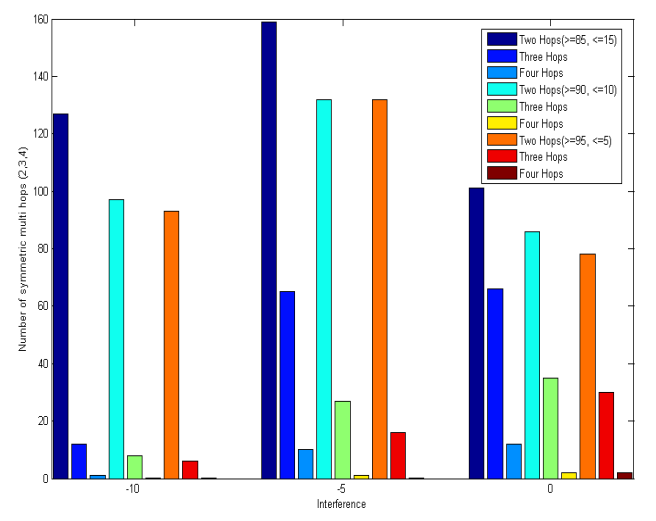

Fig. 14. Figure shows the number of two, three and four hop string topologies obtained for noise levels of $-10,-5$ and $0 \mathrm{dbm}$. For each noise level three different values of perAllowance are selected.

More complex topologies with free-space propagation characteristics are also possible. In a complex topology two nodes may be connected by one or more links (single or multi-hop). Also, two nodes may not be connected to each other. Figure 15 shows the largest topology that was extracted from the SNFI experiment with $0 \mathrm{dbm}$ noise and perAllowance set to $5 \%$. The six nodes in the topology are selected so that all links are symmetric 'up' or 'down' links (using a PERAllowance of $5 \%$ ). All nodes with asymmetric or partial connectivity were eliminated. Any two nodes may not be connected to each other. Table III shows the number of distinct topologies obtained. Topologies consisting of four to six nodes were found.

\section{Discussion}

The results show that multi-hop topologies can be successfully created with only four noise sources. Given the limited number of sources, the SNFI approach appears preferable,

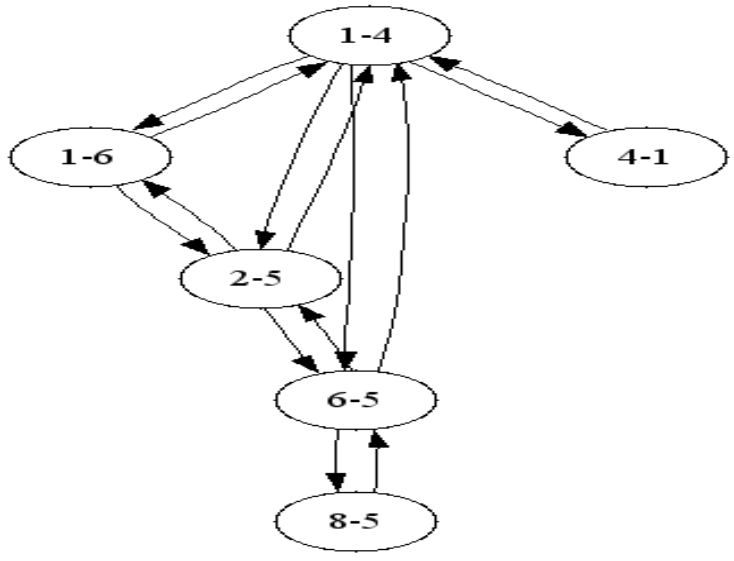

Fig. 15. Figure shows the largest symmetric topology for 0dbm noise and perAllowance set to $5 \%$. Note that the graph only indicates connectivity of nodes, it does not imply relative position amongst them.

\begin{tabular}{|l|l|l|l|l|}
\hline Size of topology & 3 & 4 & 5 & 6 \\
\hline Number of occurrences & 276 & 339 & 131 & 11 \\
\hline
\end{tabular}

TABLE III

THE NUMBER OF DIFFERENT SIZED TOPOLOGIES OBTAINED AT ODBM, PERALLOWANCE IS SET TO $5 \%$.

because it is more flexible and no manual intervention is necessary. In our setup, it can automatically select suitable nodes for a number of different 3- or 4-hop topologies. It was also possible to combine some of them into more complex topologies. While the SIFN approach could also be fully automated through an, albeit expensive, variable attenuator for each antenna, this approach will likely be limited to the nodes next to the noise sources. The availability of many noise sources would reduce this disadvantage, however.

The results also show that long-term reproducibiltiy of results is reduced if nodes with marginal SNR are involved. Simply configuring the same noise level days later is not sufficient to guarantee the same result. While this is also the case in real-world wireless experiments, a controlled testbed should aim for increased reproducibility. This problem could be addressed by executing the mapping algorithm before each experiment. The algorithm then may map the topology onto a different set of nodes. However, the PER characteristics of these nodes will be similar to the earlier experiments, which leads to improved reproducability. An open question is which of the radio mapping methods leads to better reproducibility of results. SIFN may provide more fine-grained control and thus allow a more exact reproduction of the desired PER rates between nodes, even if the radio environment has slightly changed.

The results shown in Section V hold for a beacon packet size, a bit-rate of $1 \mathrm{Mbps}$ and modulation type DBPSK/DSSS. The results may vary if any of the above mentioned parameters are changed as PER depends on the above parameters. Figure 16 shows the obtained PER at packet sizes of 32bytes and 1032 bytes at $802.11 \mathrm{~g}$ PHY rates of $1 \mathrm{Mbps}, 11 \mathrm{Mbps}$ and 


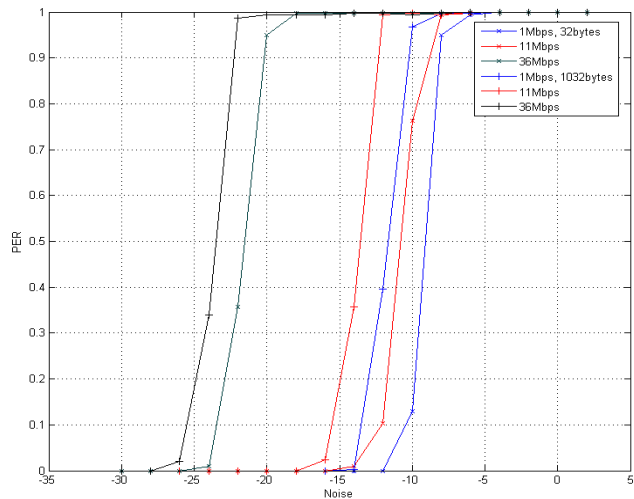

Fig. 16. PER vs Noise for packet sizes of 32 bytes and 1032 bytes at PHY rates of $1 \mathrm{Mbps}, 11 \mathrm{Mbps}$ and $36 \mathrm{Mbps}$

$36 \mathrm{Mbps}$. At any given PHY rate the change of packet size leads to a change in the PER, which can be compensated by approximately $3 \mathrm{dbm}$ change in the noise level. Similarly, the change in PER between the PHY rates of $1 \mathrm{Mbps}$ and $11 \mathrm{Mbps}$ can be compensated by upto $3 \mathrm{dbm}$ change in noise. A significant difference is seen at the PHY rate of $36 \mathrm{Mbps}$ where high PERs are observed at interference levels less by $10 \mathrm{dbm}$ than those at PHY rates of $1 \mathrm{Mbps}$ and $11 \mathrm{Mbps}$. To account for such differences the automated SNFI procedure could be extended to measure link PER for different packet sizes and modulations, and match these to a PER matrix that also includes modulation and packet size information for each PER value.

\section{CONCLUSION}

This paper described an approach to configure the placement and transmission power of additive white Gaussian noise sources to create multi-hop wireless network topologies in a constrained indoor space. In particular, the mechanisms aimed at creating topologies that match a desired PER matrix. We evaluated these algorithms by mapping a free-space propagation environment onto our testbed, which is useful in migrating protocols that from network simulators to the testbed. This approach allows isolating effects of different propagation environments from the effects of the protocol stack implementation during testing. Specifically, we conclude that

- A $20 \mathrm{MHz}$ additive white Gaussian noise signal on the 802.11 channel center frequency can effectively reduce SNR at the receiver. This can be observed from the received signal strength indicator and through increased packet error rates.

- 4-hop topologies with symmetric channels can be created with four noise sources in an $8 \mathrm{~m}$ by $8 \mathrm{~m}$ area, by placing one noise source next to each radio that is part of the experiment. They can be created more efficiently through an automatic search procedure that selects nodes whose link PER approximates the desired link qualities.
- Network and MAC-layer experiments that include nodes with marginal SNR exhibit some variability in topology when repeated after longer time periods (e.g., 24 hours), they do not show this variability over short time intervals. Better reproducibility could be achieved through an automatic calibration procedure that seeks to recreate a similar topology.

This work leads to three promising ideas for future work. First, many interesting experiments involve more than a few nodes. Thus these methods must scale to hundreds of nodes and low-cost noise sources. Second, the system could provide an improved interface that let's users specify desired topologies taking into account different modulation schemes, carrier sensing ranges, and packet size distributions. Finally, indoor testbeds should provide time-varying channel conditions that are similar to common real world deployments of wireless networks such as an office environment. Mapping time-varying scenarios onto the testbed through noise generation remains an open challenge.

\section{REFERENCES}

[1] J. T. Kaba and D. R. Raichle, "Testbed on a desktop: strategies and techniques to support multi-hop manet routing protocol development," in ACM MobiHoc. ACM Press, 2001, pp. 164-172.

[2] J. Lei, R. Yates, L. Greenstein, and H. Liu, "Wireless link snr mapping onto an indoor testbed," in IEEE Tridentcom, Feb 2005.

[3] J. Bicket, D. Aguayo, S. Biswas, and R. Morris, "Architecture and evaluation of an unplanned 802.11b mesh network,' in MobiCom '05: Proceedings of the 11th annual international conference on Mobile computing and networking. New York, NY, USA: ACM Press, 2005, pp. $31-42$.

[4] D. Kotz and K. Essien, "Analysis of a campus-wide wireless network," in Proceedings of the eighth annual international conference on Mobile computing and networking. ACM Press, 2002, pp. 107-118.

[5] D. Raychaudhuri, I. Seskar, M. Ott, S. Ganu, K. Ramachandran, H. Kremo, R. Siracusa, H. Liu, and M. Singh, "Overview of the ORBIT radio grid testbed for evaluation of next-generation wireless network protocols," in IEEE WCNC, 2005.

[6] K. Papagiannaki, M. Yarvis, and W. S. Conner, "Experimental characterization of home wireless networks and design implications," in INFOCOM, 2006 (to be printed). [Online]. Available: http: //www.cambridge.intel-research.net/ kpapagia/papers/homenet.pdf

[7] W. Ye, "The ns manual, ch 18: Radio propagation models," http://www. isi.edu/nsnam/ns/doc/index.html.

[8] S. Sanghani, T. Brown, S. Bhandare, and S. Doshi, "EWANT: The emulated wireless ad hoc network testbed," in IEEE WCNC, vol. 3, Mar 2003, pp. 1844-1849.

[9] K. Pajukoski and J. Savusalo, "Wideband CDMA test system," in Symposium on Personal Indoor and Mobile Radio Communications, 1997.

[10] D. Kotz, C. Newport, R. S. Gray, J. Liu, Y. Yuan, and C. Elliott, "Experimental evaluation of wireless simulation assumptions," in ACM/IEEE MSWiM, October 2004, pp. 78-82. [Online]. Available: http://www.cs.dartmouth.edu/ dfk/papers/kotz:axioms.pdf

[11] H. Lundgren, D. Lundberg, J. Nielsen, E. Nordstrom, and C. Tschudin, "A large-scale testbed for reproducible ad hoc protocol evaluation," in IEEE WCNC, 2002.

[12] D. Maltz, J. Broch, and D. Johnson, "Experiences designing and building a multi-hop wireless ad hoc network testbed," Carnegie Mellon University, Tech. Rep. CMU-CS-99-116, Mar 1999.

[13] M. Möske, H. Füssler, H. Hartenstein, and W. Franz, "Performance measurements of a vehicular ad hoc network," in Vehicular Technology Conference (VTC-Spring), May 2004, pp. 2116-2120.

[14] E. Kohler, R. Morris, B. Chen, J. Jannotti, and M. F. Kaashoek, "The click modular router,' ACM Transactions on Computer Systems, vol. 18, no. 3, pp. 263-297, Aug 2000. 